\title{
On the maximal diphoton width
}

\author{
Alberto Salvio, ${ }^{a}$ Florian Staub, ${ }^{a}$ Alessandro Strumia ${ }^{a, b}$ and Alfredo Urbano ${ }^{a}$ \\ a Theoretical Physics Department, CERN, \\ Geneva, Switzerland \\ ${ }^{b}$ Dipartimento di Fisica, Università di Pisa and INFN, \\ largo Bruno Pontecorvo 3, Pisa, Italy \\ E-mail: alberto.salvio@cern.ch, florian.staub@cern.ch, \\ astrumia@cern.ch, alfredo.leonardo.urbano@cern.ch
}

ABSTRACT: Motivated by the $750 \mathrm{GeV}$ diphoton excess found at LHC, we compute the maximal width into $\gamma \gamma$ that a neutral scalar can acquire through a loop of charged fermions or scalars as function of the maximal scale at which the theory holds, taking into account vacuum (meta)stability bounds. We show how an extra gauge symmetry can qualitatively weaken such bounds, and explore collider probes and connections with Dark Matter.

Keywords: Beyond Standard Model, Renormalization Group, Higgs Physics

ArXIV EPRINT: 1602.01460 


\section{Contents}

1 Introduction 1

2 A fermionic loop $\quad 2$

3 A scalar loop $\quad 4$

3.1 A single charged scalar 4

3.1.1 Meta-stability 5

3.1.2 Perturbativity limits 6

3.2 Many scalars 8

3.2.1 Perturbativity limits $\quad 8$

3.2.2 Gauged $\mathrm{SU}(N) \quad 9$

4 Collider probes and dark matter $\quad 10$

$\begin{array}{lll}4.1 & \text { Collider probes } & 10\end{array}$

$\begin{array}{lll}4.2 & \text { Dark matter } & 11\end{array}$

4.3 Precision observables 12

5 Conclusions 13

\section{Introduction}

The ATLAS and CMS collaborations found an excess in $p p \rightarrow \gamma \gamma$ events $[1,2]$ that can be interpreted as the production of a new scalar resonance $S$ with mass $M_{S} \approx 750 \mathrm{GeV}$, provided that $S$ has a large enough width into photons, $\Gamma_{\gamma \gamma}=\Gamma(S \rightarrow \gamma \gamma)$. Assuming that $S$ is produced trough $g g$ or $q \bar{q}$ partonic collisions, the claimed $\gamma \gamma$ excess can be reproduced for $\Gamma_{\gamma \gamma} / M_{S} \approx 10^{-6}$ if the $S$ width is narrow, and for $\Gamma_{\gamma \gamma} / M \approx 10^{-4}$ if the total width is large, $\Gamma_{S} \sim 0.06 M_{S}$. Larger values $\Gamma_{\gamma \gamma} / M \approx 10^{-3}$ are needed if $S$ is produced trough $\gamma \gamma$ partonic collisions [3-6].

This raises a theoretical question: how can such a width be obtained in a fundamental theory? Extra charged fermions or scalars $X$ must be present to mediate the $S \rightarrow \gamma \gamma$ process, and they must be coupled to $S$, through Yukawa couplings $y$ or scalar cubic couplings $\kappa$.

In the fermionic case, $\Gamma_{\gamma \gamma}$ gets enhanced by considering a large Yukawa $y$ and/or a large multiplicity $N$ and/or a large hypercharge $Y$ of the new fermions. All these enhancements imply that some coupling ( $y$ and/or $g_{Y}$ ), when renormalised up to higher energies, becomes larger until it develops a Landau pole, signalling the presence of new non-perturbative physics $[3,7,8]$. In section 2 we revisit such issues, adding the extra constraint of vacuum stability along the $S$ direction. 
$\Gamma(S \rightarrow \gamma \gamma)$ from a fermion loop, $M_{\psi}=375 \mathrm{GeV}$

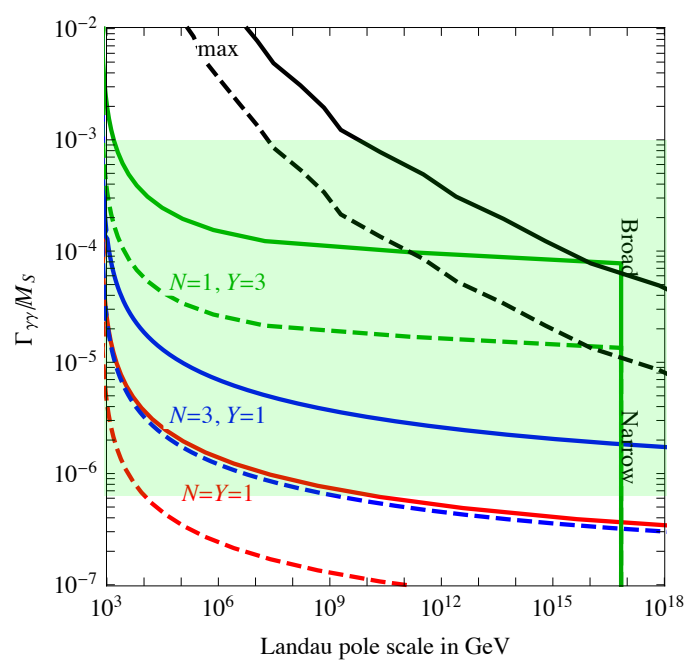

$\Gamma(S \rightarrow \gamma \gamma)$ from a fermion loop, $M_{\psi}=1 \mathrm{TeV}$

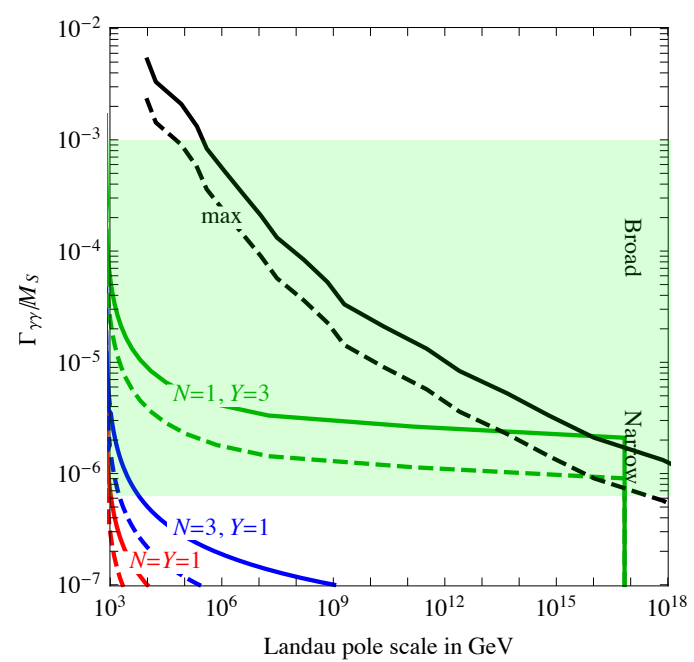

Figure 1. Maximal $\Gamma_{\gamma \gamma}$ generated by a fermionic loop compatible with perturbativity considering a $750 \mathrm{GeV}$ scalar (dashed curves) or pseudo-scalar (continuous curves) with a CP-conserving Yukawa coupling. The green band shows the value of $\Gamma_{\gamma \gamma}$ favored by the $750 \mathrm{GeV}$ excess, assuming that $S$ has a narrow (lower) or broad (upper) width.

In the scalar case, the loop that mediates $S \rightarrow \gamma \gamma$ can be enhanced by a large cubic $\kappa S|X|^{2}$ [9-11]. At first sight, this presents two possible advantages. First, the RGE evolution of $\kappa$ never generates Landau poles since it has dimension 1 and thereby corresponds to a relevant operator (unlike the dimensionless Yukawa coupling $y$ introduced in the fermionic case). Furthermore, a large cubic can arise if there is a weakly-coupled ${ }^{1}$ scalar sector around $\approx 10 \mathrm{TeV}$ that contains the light scalars $S$ and $X$ with a cubic coupling among them, which does not get suppressed. However, a large cubic leads to extra minima in the potential $V(S, X)$ and is thereby subject to vacuum stability bounds. In this work we consider absolute stability and meta-stability. We will find that, after imposing such bounds, the maximal $\Gamma_{\gamma \gamma}$ given by a scalar loop is similar to the maximal $\Gamma_{\gamma \gamma}$ produced by a fermion loop.

In section 2 we reconsider fermion models. In section 3 we consider scalar models. Signals at colliders and connection with Dark Matter is discussed in section 4. Conclusions are given in section 5 .

\section{A fermionic loop}

We couple $S$ to $N$ fermions $\psi$ with mass $M_{\psi}$, hypercharge $Q=Y$ and singlet under $\operatorname{SU}(2)_{L}$. We assume that the $N$ fermions have the same mass and same couplings, such that the Lagrangian

$$
\mathscr{L}=\mathscr{L}_{\mathrm{SM}}+\frac{\left(\partial_{\mu} S\right)^{2}}{2}+\bar{\psi}\left(i \not D-M_{\psi}\right) \psi+\left[S \bar{\psi}\left(y+i \tilde{y} \gamma_{5}\right) \psi+\text { h.c. }\right]-V(S)-V(S, H)
$$

\footnotetext{
${ }^{1}$ The weak-coupling ensures computability.
} 
respects a $\mathrm{SU}(N)$ symmetry, under which $\psi$ is in the fundamental representation: this choice simplifies computations and maximises $\Gamma_{\gamma \gamma}$. The potential is $V(S)=\frac{1}{2} M_{S}^{2} S^{2}+\lambda_{S} S^{4}$. The Yukawa coupling $y(\tilde{y})$ is present if $S$ is a scalar (pseudo-scalar). $y$ and $\tilde{y}$ have the same RGE, and $\tilde{y}$ contributes more to $S \rightarrow \gamma \gamma$ than $y$ (see e.g. [3]). If $S$ is a pseudo-scalar the loop function is maximal at $M_{\psi}=M_{S} / 2$, and we find

$$
\left.\frac{\Gamma_{\gamma \gamma}}{M} \approx 0.610^{-6} N^{2} \tilde{y}^{2} Y^{4}\right|_{M_{\psi}=M_{S} / 2},\left.\quad \frac{\Gamma_{\gamma \gamma}}{M} \approx 0.210^{-9} N^{2} \tilde{y}^{2} Y^{4}\right|_{M_{\psi}=1 \mathrm{TeV}}
$$

The formula for general $M_{\psi}$ can be found in ref. [3]. Allowing $\mathrm{SU}(N)$ to become a gauge symmetry (we will see below why this is useful) with gauge constant $g$, the relevant RGE are

$$
\begin{aligned}
(4 \pi)^{2} \beta_{g_{Y}} & =g_{Y}^{3}\left(\frac{41}{6}+\frac{4 N}{3} Y^{2}\right) \\
(4 \pi)^{2} \beta_{g} & =-b g^{3} \quad b=\frac{11}{3} N-\frac{2}{3}-\cdots \\
(4 \pi)^{2} \beta_{y} & =(2 N+3) y^{3}-y\left(6 g_{Y}^{2} Y^{2}+3 \frac{N^{2}-1}{N} g^{2}\right) \\
(4 \pi)^{2} \beta_{\lambda_{S}} & =72 \lambda_{S}^{2}+2 N y^{2}\left(4 \lambda_{S}-y^{2}\right)
\end{aligned}
$$

where $\beta_{\theta} \equiv d \theta / d \ln \mu$ and $\cdots$ denotes the contribution of extra possible particles charged under $\mathrm{SU}(N)$. For simplicity, we assumed a vanishing quartic coupling $|S|^{2}|H|^{2}{ }^{2}$

Assuming $g=0$ and ignoring eq. (2.3d) we reproduce the results of $[3,7,8]$, that we plot in figure 1 as the maximal value of $\Gamma_{\gamma \gamma}$ as function of the Landau poles scale. We have checked that taking into account the RGE for $\lambda_{S}$, eq. (2.3d), which was partially considered in [12-15], does not affect these results.

We impose that $\lambda_{S}$ does not hit a Landau pole, and that it does not lead to too fast vacuum decay $[17,18]$ : this gives the upper and lower bound in the following formula, respectively

$$
-\frac{0.016}{1+0.01 \ln \mu / M_{S}}<\lambda_{S}(\mu) \lesssim 4 \pi
$$

Combining eq. (2.2) with eq. (2.3a) shows that the maximal $\Gamma_{\gamma \gamma}$ is obtained for small $N=1$ and for $Y$ as large as allowed by Landau poles for hypercharge, which corresponds to uninteresting values $Y \sim 10$. Thereby, we also plotted the maximal $\Gamma_{\gamma \gamma}$ at fixed values of $Y$ and $N$. We see that $\Gamma_{\gamma \gamma} \lesssim 10^{-6}$ can be obtained within models with reasonable $Y \sim 1$ and $N \lesssim 3$ that remain perturbative up to the Planck scale. Larger values of $\Gamma_{\gamma \gamma}$ need new non-perturbative physics not much above the $\mathrm{TeV}$ scale, especially if the fermion $\psi$ is colored, such that it can also mediate $S \rightarrow g g$ but needs to be heavier of about $1 \mathrm{TeV}$ in view of LHC bounds.

Gauged SU(N). Finally, we consider the new class of models obtained gauging $\mathrm{SU}(N)$. Such models interpolate between weakly-coupled models $(g=0)$ and strongly-coupled models $\left(g\right.$ becomes non perturbative around $M_{S}$ ) considered in the literature [3, 19-24]. It is interesting to notice that, even without considering the non-perturbative limit, a

\footnotetext{
${ }^{2}$ This coupling was considered in [12-15] and helps in stabilising the electroweak (EW) vacuum [16].
} 
perturbative $g$ allows to obtain qualitatively larger values of $y$ and thereby of $\Gamma_{\gamma \gamma}$ without hitting Landau poles than in the $g=0$ limit. Indeed, if $g>0$, the RGE for $y$, eq. (2.3c), implies that the low energy value of $y$ is attracted towards the Pendleton-Ross infra-red fixed point [25-28]

$$
\frac{y^{2}}{g^{2}} \rightarrow \frac{3(N-1 / N)-b}{2 N+3}
$$

provided that the latter term is positive, $b<3(N-1 / N)$. In such a case, $y$ at low energy can become arbitrarily large without hitting Landau poles, given that the same holds for $g$. For example, in the limit of large $N$ and small $b$ one has $y^{2} / g^{2} \rightarrow 3 / 2$.

If instead the latter term in eq. (2.5) is negative the infra-red fixed point does not exist, and adding a $g>0$ does not give a result qualitatively different from in the $g=0$ limit.

\section{A scalar loop}

We now consider the scalar case, which requires discussing the (meta)stability of the full potential. Thereby we first consider the case of a single scalar.

\subsection{A single charged scalar}

We start considering the following minimal model, where the SM is extended by adding a neutral real scalar singlet $S$ and one complex singlet $X$ with hypercharge $Y=Q$. The scalar potential is

$$
V(H, S, X)=-\frac{M_{h}^{2}}{2}|H|^{2}+\lambda_{H}|H|^{4}+\lambda_{H S}|H|^{2} S^{2}+\lambda_{H X}|H|^{2}|X|^{2}+\kappa_{H S} S|H|^{2}+V(S, X)
$$

where the terms involving only the new scalars $S$ and $X$ are

$$
V(S, X)=\frac{M_{S}^{2}}{2} S^{2}+M_{X}^{2}|X|^{2}+\lambda_{S} S^{4}+\lambda_{X S} S^{2}|X|^{2}+\lambda_{X}|X|^{4}+\frac{\kappa_{S}}{3} S^{3}+\kappa_{X S} S|X|^{2}
$$

At very large field values (when $S$ and $X$ are much bigger than the dimensionfull parameters) the potential is stable if $\lambda_{S}, \lambda_{X}>0$ and $\lambda_{X S}^{2}<4 \lambda_{S} \lambda_{X}$ [16]. The resulting $S$ width into photons is

$$
\frac{\Gamma(S \rightarrow \gamma \gamma)}{M}=\frac{\alpha_{\mathrm{em}}^{2}}{256 \pi^{3}}\left|\frac{\kappa_{X S} M_{S}}{2 M_{X}^{2}} Q^{2} F\left(\frac{4 M_{X}^{2}}{M_{S}^{2}}\right)\right|^{2}
$$

where the loop function $F$ is

$$
F(x)=x\left[x \arctan ^{2}\left(\frac{1}{\sqrt{x-1}}\right)-1\right] \stackrel{x \rightarrow \infty}{=} \frac{1}{3} .
$$

Considering the potential as function of $S$ only, absolute stability is satisfied for $\left|\kappa_{S}\right|^{2}<$ $18 M_{S}^{2} \lambda_{S}$. In the presence of both $S$ and $X$, absolute stability can be again computed analytically, although the result is too long to be presented. The main qualitative feature is that the upper bound on $\Gamma(S \rightarrow \gamma \gamma) \propto\left|\kappa_{X S}\right|^{2}$ grows proportionally to some combination linear in the quartics $\lambda_{S}, \lambda_{X S}, \lambda_{X}$. This means that the scalar loop contribution to $S \rightarrow \gamma \gamma$ is limited by perturbativity of the quartics, just like a fermion loop contribution is limited by perturbativity of the Yukawa $y^{2}$. Then our goal is generalising to scalar case the result found in the fermionic case and shown in figure 1. 

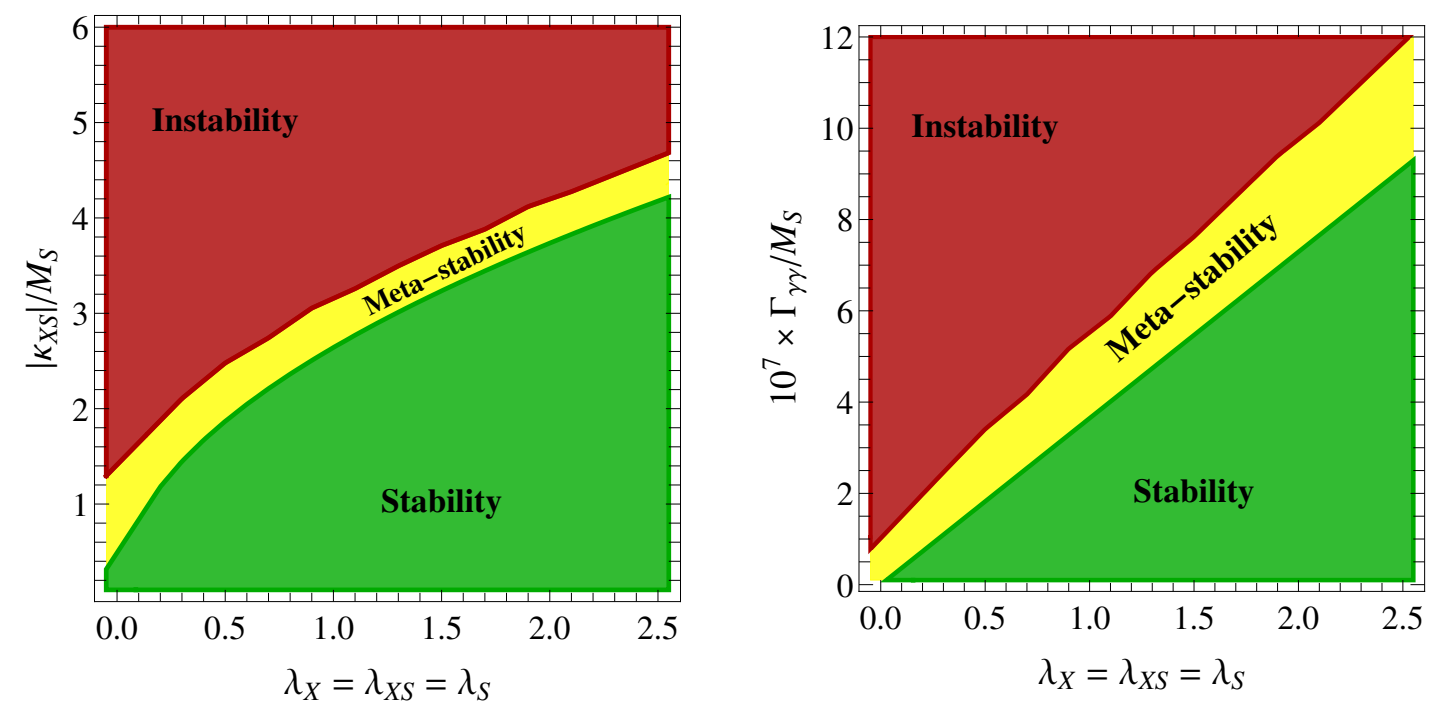

Figure 2. Left (a): maximal cubic $\left|\kappa_{X S}\right| / M_{S}$ allowed by stability (green) and by meta-stability (yellow) as function of $\lambda_{X}=\lambda_{S}=\lambda_{X S}$. Right (b): the corresponding value of $\Gamma_{\gamma \gamma} / M$ assuming that the scalar $X$ has charge $Q=1$. Vacuum decay is too fast in the red regions.

\subsubsection{Meta-stability}

The meta-stability condition can be computed only numerically, and is weaker than the stability condition, altought they are qualitatively similar.

For the numerical computation we use the tool-chain SARAH-SPheno-Vevacious: we implemented the minimal model of eq. (3.2) in SARAH [29-34] and generated the Fortran code for SPheno $[35,36]$ to get a spectrum generator for the model. SPheno was used to compute all masses and branching ratios, and the produced spectrum file is then given to Vevacious [37] as input to check the stability of the electroweak vacuum. For this purpose, we generated a model file with SARAH for Vevacious which includes the possibility of VEVs for the charged scalar beside to ones for the neutral states. Vevacious checks the stability of the scalar potential via a homotopy method which guarantees to find all minima of the tree-level potential. In the case that there is a minimum deeper than the desired one, it calls ComsoTransitions [38] to calculate the life-time of the vacuum. The decay rate $\Gamma$ per unit volume for false vacuum decay can be written as $[17,18]$

$$
\frac{d \wp}{d V d t}=\frac{e^{-S}}{R^{4}}
$$

where $R \approx 1 / M_{S}$ is the size of the bounce and $S$ is the action of the bounce. At tree level it is given by

$$
S=\int d^{4} x\left(\frac{\left(\partial_{\mu} S\right)^{2}}{2}+\left|D_{\mu} X\right|^{2}+V(S, X)\right) .
$$

CosmoTransitions finds the multi-field optimal 'path' to tunnel from the false to the true vacuum using the B-splines algorithm. For more technical details we refer to [38]. 
Integrating over our past-light cone, taking into account the expansion of the universe, we find the present value of the vacuum-decay probability $\wp$,

$$
\wp_{0}=0.15 \frac{e^{-S}}{\left(R H_{0}\right)^{4}},
$$

where $H_{0} \approx 67.4 \mathrm{~km} / \mathrm{sec} \mathrm{Mpc}$ is the present Hubble rate. A probability $\wp_{0}$ larger than $10 \%$ is obtained for $S>412$. Based on the result of this calculation, we label as meta-stable a point such that $\wp_{0}>10 \%$, and unstable otherwise.

To start and to illustrate the result, we consider the special case $\lambda_{X}=\lambda_{X S}=\lambda_{S}$ and $\kappa_{S}=0$. Furthermore we fix $M_{X}=M_{S} / 2$, which is the value that maximises $\Gamma_{\gamma \gamma}$. Figure 2a shows the resulting stability region (green): the maximal $\left|\kappa_{X S}\right|$ grows proportionally to the squared root of the couplings. The extra region allowed by meta-stability (in yellow) has a similar shape. In figure $2 \mathrm{a}$ we show the corresponding $\Gamma_{\gamma \gamma}$ rate, assuming a single scalar $X$ with $Q=1$ : we see that a phenomenologically relevant value $\Gamma_{\gamma \gamma} \gtrsim 10^{-6}$ needs quartic couplings of order 1.

\subsubsection{Perturbativity limits}

In order to quantify if a TeV-scale value of the quartics is 'too large', we solve their oneloop renormalisation group equations and compute the RGE energy scale $\mu=\Lambda$ at which a coupling hits a Landau pole. The RGEs that involve only the quartic couplings of $S, X$ are (the full set of RGE is given later)

$$
\begin{aligned}
\beta_{\lambda_{X}} & =\frac{1}{(4 \pi)^{2}}\left[20 \lambda_{X}^{2}+2 \lambda_{X S}^{2}\right] \\
\beta_{\lambda_{X S}} & =\frac{1}{(4 \pi)^{2}}\left[8 \lambda_{X S}^{2}+8 \lambda_{X S}\left(\lambda_{X}+3 \lambda_{S}\right)\right] \\
\beta_{\lambda_{S}} & =\frac{1}{(4 \pi)^{2}}\left[\lambda_{X S}^{2}+72 \lambda_{S}^{2}\right] .
\end{aligned}
$$

A large coupling leads to a Landau pole at low energy; in such a case $\Lambda$ can be approximated as

$$
\Lambda \approx M_{S} \exp \min _{\lambda} \frac{\lambda}{\beta_{\lambda}}
$$

which becomes exact in the case of a single quartic coupling.

We next perform a full scanning, picking $10^{4}$ random points in the 4-dimensional parameter space of the model, and checking if stability and/or meta-stability are satisfied; in such a case we compute $\Gamma_{\gamma \gamma}$ and the Landau pole scale. The final result is shown in figure 3: like in the fermionic case, a larger $\Gamma_{\gamma \gamma}$ implies a Landau pole at lower energy. Actually, the maximal $\Gamma_{\gamma \gamma}$ is a factor of few lower than in the corresponding fermionic case.

The $\Gamma_{\gamma \gamma}$ width can be increased by allowing for a scalar $X$ with bigger charge and/or for multiple states $X$. However, these possibilities are limited by Landau poles for the hypercharge gauge coupling and by precision data, as studied in the next section. 

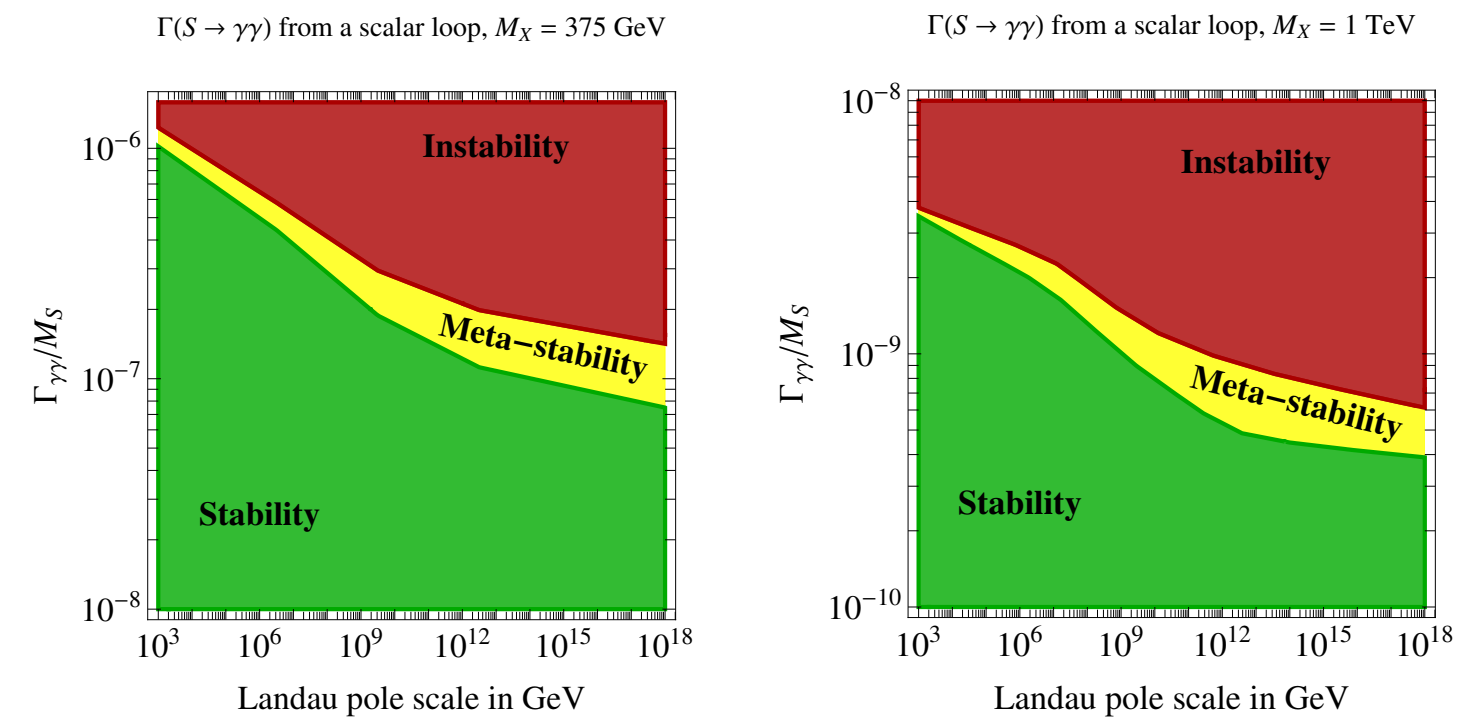

Figure 3. Maximal $\Gamma_{\gamma \gamma}$ allowed by perturbativity considering a scalar $S$ with a cubic coupling to one singlet charged scalar $X$ with $Q=Y=1$.
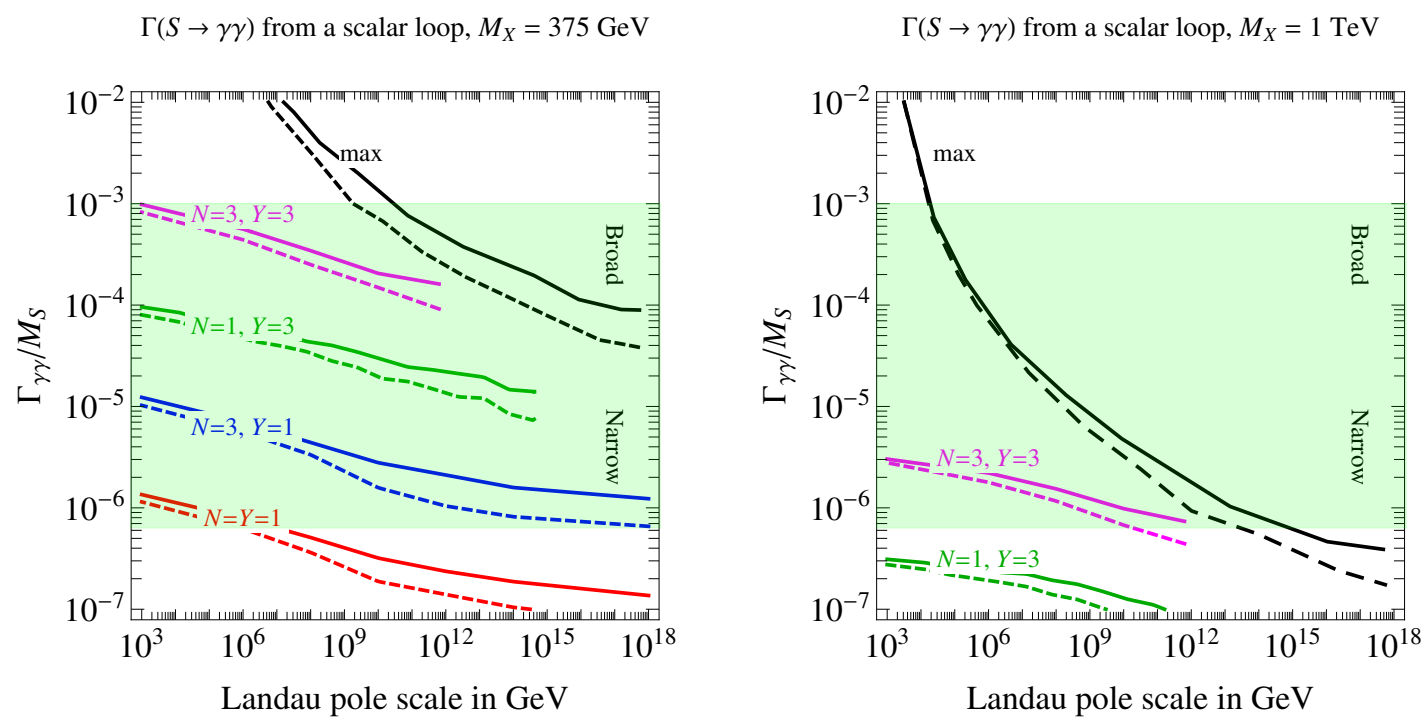

Figure 4. Maximal $\Gamma_{\gamma \gamma}$ generated by a scalar loop compatibly with vacuum stability (dashed curves) or by meta-stability (continuous curves) as function of the scale at which the theory becomes non-perturbative. The upper curves in black refer to a generic set of scalars; the lower curves to some special case: a single scalar $(N=1)$ with unity hypercharge $(Y=1)$, multiple fields (blue, $N=3$ ), bigger hypercharge (green, $Y=3$ ) and both (magenta, $N=Y=3$ ). The maximal $\Gamma_{\gamma \gamma}$ is obtained for $M_{X}=M_{S} / 2$ (left panel); in the right panel we consider $M_{X}=1 \mathrm{TeV}$, which is allowed by LHC data if the scalar fields are colored. 


\subsection{Many scalars}

We now generalize the results of the previous section including more charged scalars. We consider $N$ scalars $X$ with hypercharge $Y$ and singlet under $\mathrm{SU}(2)_{L}$, assumed to lie in a fundamental representation of an extra $\mathrm{SU}(N)$ global or gauge symmetry. This means that all scalars have the same mass and the same cubic: this choice maximises their effect on $\Gamma_{\gamma \gamma}$ for a fixed scale at which the theory becomes non-perturbative. The vacuum stability and meta-stability conditions remain the same as in the previous section: we just need to take into account the enhancement in $\Gamma_{\gamma \gamma}$ and the modified perturbativity conditions.

\subsubsection{Perturbativity limits}

We write the RGE including all relevant SM couplings: the gauge couplings $g_{3}, g_{2}$ and $g_{1} \equiv \sqrt{5 / 3} g_{Y}$, the top Yukawa coupling $y_{t}$ and the quartic couplings $\lambda_{H}, \lambda_{H S}$ and $\lambda_{H X}$. We also consider the (possibly vanishing) gauge coupling of $\mathrm{SU}(N), g$. The RGEs for the dimensionless couplings $\lambda_{X}, \lambda_{X S}, \lambda_{S}, g_{1}$ and $g$ are

$$
\begin{aligned}
(4 \pi)^{2} \beta_{\lambda_{X}}= & 4(N+4) \lambda_{X}^{2}+2 \lambda_{X S}^{2}-\frac{36 Y^{2} g_{1}^{2} \lambda_{X}}{5}+\frac{54 Y^{4}}{25} g_{1}^{4}+2 \lambda_{H X}^{2}+ \\
& +\frac{3(N-1)\left(N^{2}+2 N-2\right)}{4 N^{2}} g^{4}-\frac{6\left(N^{2}-1\right)}{N} g^{2} \lambda_{X}, \\
(4 \pi)^{2} \beta_{\lambda_{X S}}= & 8 \lambda_{X S}^{2}+4 \lambda_{X S}\left[(1+N) \lambda_{X}+6 \lambda_{S}-\frac{9 Y^{2} g_{1}^{2}}{10}\right]+ \\
& +4 \lambda_{H S} \lambda_{H X}-\frac{3\left(N^{2}-1\right)}{N} \lambda_{X S} g^{2} \\
(4 \pi)^{2} \beta_{\lambda_{S}}= & N \lambda_{X S}^{2}+72 \lambda_{S}^{2}+2 \lambda_{H S}^{2}, \\
(4 \pi)^{2} \beta_{g_{1}}= & g_{1}^{3} \frac{41+2 N Y^{2}}{10}, \\
(4 \pi)^{2} \beta_{g}= & g^{3}\left(-\frac{11}{3} N+\frac{1}{6}\right) .
\end{aligned}
$$

We included the quartic couplings $\lambda_{H S}$ and $\lambda_{H X}$ that involve the Higgs boson because, although they negligibly affect the non-perturbativity issue, they unavoidably enter into the RGEs for $\lambda_{X}, \lambda_{X S}, \lambda_{S}$. Indeed the quartic $\lambda_{H X}$ is unavoidably generated by hypercharge interactions because both $H$ and $X$ are charged; then a $\lambda_{H S}$ coupling is generated too as dictated by the following RGEs:

$$
\begin{aligned}
(4 \pi)^{2} \beta_{\lambda_{H X}}= & \lambda_{H X}\left[4(1+N) \lambda_{X}-\frac{\left(36 Y^{2}+9\right) g_{1}^{2}}{10}-\frac{9 g_{2}^{2}}{2}+12 \lambda_{H}+6 y_{t}^{2}\right] \\
& +4 \lambda_{H S} \lambda_{X S}+4 \lambda_{H X}^{2}+\frac{27 g_{1}^{4} Y^{2}}{25}-\frac{3\left(N^{2}-1\right)}{N} \lambda_{H X} g^{2} \\
(4 \pi)^{2} \beta_{\lambda_{H S}}= & 2 N \lambda_{X S} \lambda_{H X}+8 \lambda_{H S}^{2}+\lambda_{H S}\left(24 \lambda_{S}-\frac{9 g_{1}^{2}}{10}-\frac{9 g_{2}^{2}}{2}+6 y_{t}^{2}+12 \lambda_{H}\right) \\
(4 \pi)^{2} \beta_{\lambda_{H}}= & 2 \lambda_{H S}^{2}+N \lambda_{H X}^{2}+\frac{27 g_{1}^{4}}{200}+\frac{9 g_{1}^{2} g_{2}^{2}}{20}+\frac{9 g_{2}^{4}}{8}+\lambda_{H}\left(-\frac{9 g_{1}^{2}}{5}-9 g_{2}^{2}+12 y_{t}^{2}\right) \\
& +24 \lambda_{H}^{2}-6 y_{t}^{4},
\end{aligned}
$$




$$
\begin{aligned}
& (4 \pi)^{2} \beta_{y_{t}}=y_{t}\left(\frac{9}{2} y_{t}^{2}-\frac{17 g_{1}^{2}}{20}-8 g_{3}^{2}-\frac{9 g_{2}^{2}}{4}\right) \\
& (4 \pi)^{2} \beta_{g_{2}}=-\frac{19 g_{2}^{3}}{6}, \quad(4 \pi)^{2} \beta_{g_{3}}=-7 g_{3}^{3} .
\end{aligned}
$$

We are now ready to present our final result. Setting $g=0$ (global $\mathrm{SU}(N)$ symmetry) in figure $4 \mathrm{~b}$ we show the maximal value of $\Gamma_{\gamma \gamma}$, as function of the scale at which a Landau pole develops. $\Gamma_{\gamma \gamma}$ gets significantly enhanced, even by orders of magnitude, with respect to the minimal case $N=Y=1$ considered in section 3.1. The plot also shows the special cases $N=3$ and $Q=3$. The final result is similar to the analogous fermionic result, shown in figure 1.

\subsubsection{Gauged $\mathrm{SU}(N)$}

The gauging of the $\mathrm{SU}(N)$ symmetry allows, both in the fermionic and in the scalar case, to get larger values of $\Gamma_{\gamma \gamma}$ without hitting Landau poles. Indeed, if $g$ runs becoming larger at low energy, the quartic $\lambda_{X}$ gets driven to comparably large values, being attracted towards the quasi-fixed point $[25-28]$

$$
\frac{\lambda_{X}}{g^{2}} \rightarrow \frac{s_{\lambda g}-b+\sqrt{\left(s_{\lambda g}-b\right)^{2}-4 s_{\lambda} s_{g}}}{2 s_{\lambda}}
$$

where $b, s_{g}, s_{\lambda}, s_{\lambda g}$ are constants that parameterise the RGE coefficients as

$$
(4 \pi)^{2} \beta_{g}=-b g^{3}, \quad(4 \pi)^{2} \beta_{\lambda_{X}}=2\left[s_{\lambda} \lambda_{X}^{2}-s_{\lambda g} \lambda_{X} g^{2}+s_{g} g^{4}\right],
$$

For example we find $\lambda_{X} / g^{2} \rightarrow(3+\sqrt{6}) / 4$ in the limit of large $N$ and small $b \ll s_{\lambda g}$. Like in the fermionic case, the qualitative properties of $\lambda_{X}$ from eq.s (3.13) depend on the sign of

$$
E \equiv \frac{\left(s_{\lambda g}-b\right)^{2}-4 s_{\lambda} s_{g}}{4 s_{g}^{2}}
$$

(see the last article in [25-28]). The infra-red fixed point exists if $E \geq 0$; in such a case there is no Landau pole for values of the quartic such that $\sqrt{E} \leq D \leq \Lambda_{0}+\sqrt{E}$, where

$$
D \equiv \frac{s_{\lambda g}-b}{2 s_{g}}, \quad \Lambda_{0} \equiv \frac{g^{2}\left(\mu_{0}\right)}{\lambda\left(\mu_{0}\right)}
$$

and $\mu_{0}$ is some reference energy. If instead $E<0$ there is always a Landau pole.

This situation is illustrated in figure 5 and in its caption. The plot on the right has $E \geq 0$ (so a fixed point is allowed), and the low-energy value of $y$ remains finite even assuming no Landau pole up to arbitrarily large energy. The plot on the left has $E<0$ (no fixed point), and $y$ at low energy must be small if the theory cannot have Landau poles up to higher energy: allowing a $g \neq 0$ only has a minor effect with respect to the $g=0$ limit. 

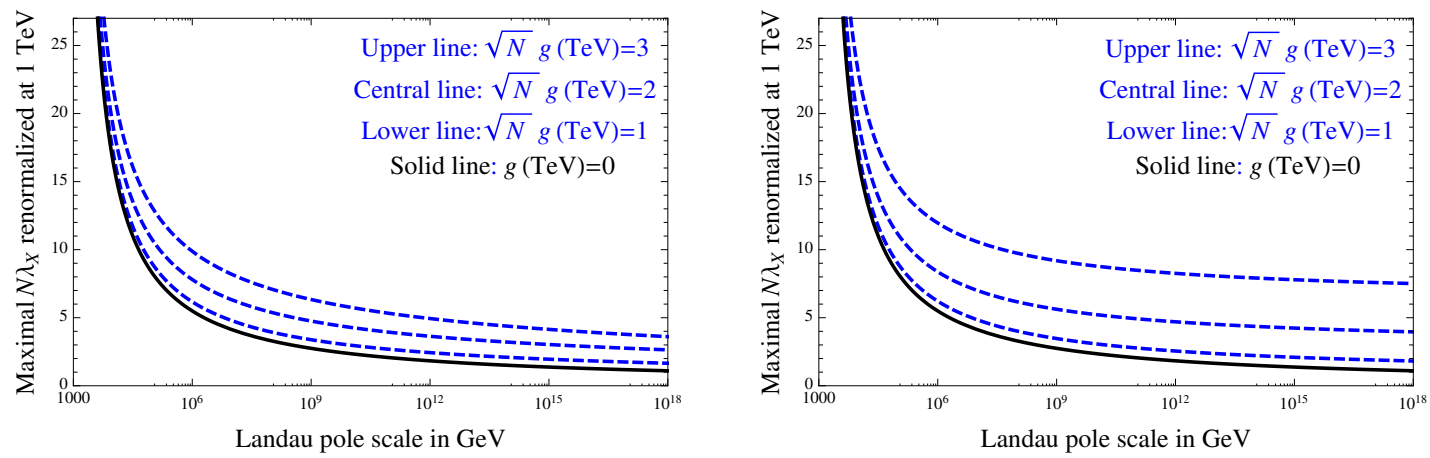

Figure 5. Maximal $N \lambda_{X}$ at low energy (chosen to be $1 \mathrm{TeV}$ ) as function of the maximal energy at which the theory holds without hitting Landau poles. We consider the large $N$ limit and fixed values of the 't Hooft coupling $\sqrt{N} g$ at TeV energy. Left: no fixed points. Right: the gauge beta function is reduced to $b=N$ such that a fixed point for $y$ arises.
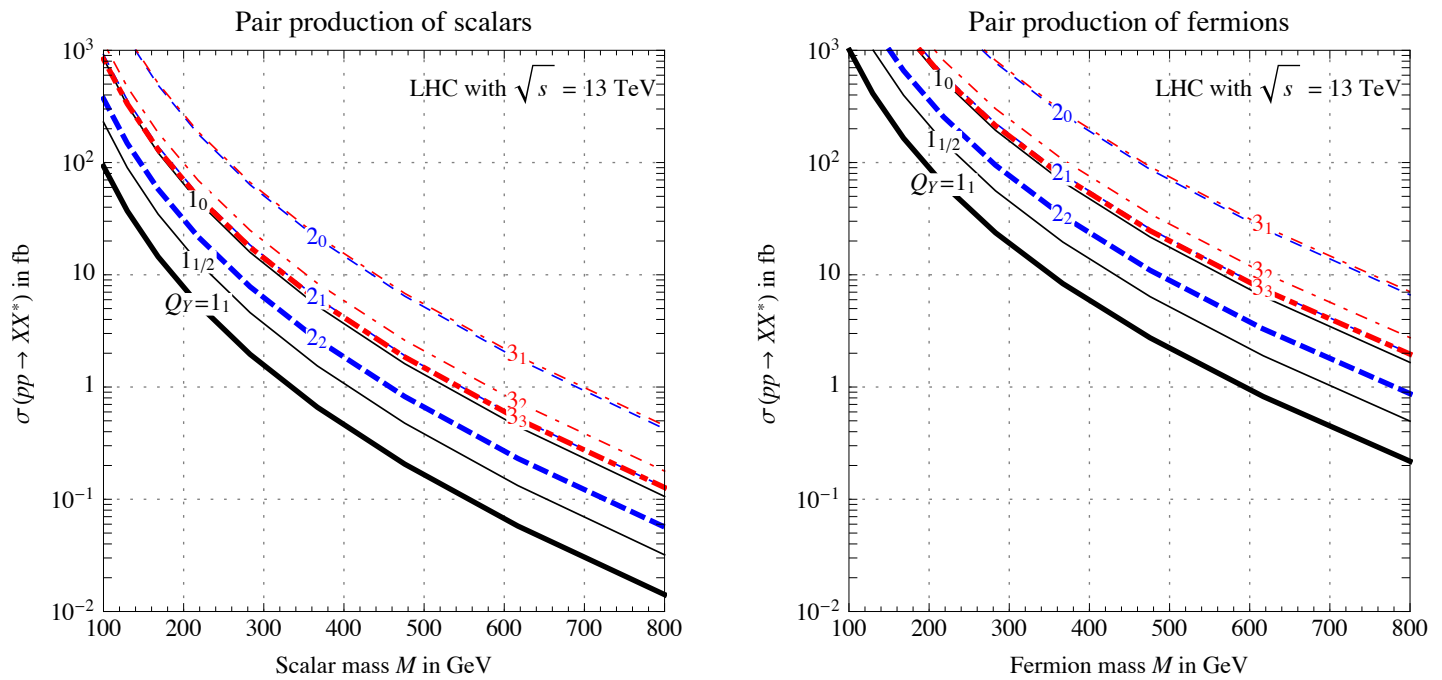

Figure 6. Cross section $p p \rightarrow X X^{*}$ for producing two uncolored particles (scalars in the left plot and fermions in the right plot) with charge $Q$ and hypercharege $Y$, indicated as $Q_{Y}$.

\section{Collider probes and dark matter}

\subsection{Collider probes}

We now discuss how the scenario can be probed at colliders. The partonic cross section $q_{1} \bar{q}_{2} \rightarrow X_{1} \bar{X}_{2}$ for pair production of two uncolored scalars or fermions $X_{1} \bar{X}_{2}$ is

$$
\frac{d \sigma}{d \hat{t}}=\frac{V_{L}^{2}+V_{R}^{2}}{144 \pi \hat{s}^{2}} \times \begin{cases}\left(2 M_{1}^{2} M_{2}^{2}+\hat{s}^{2}-2\left(M_{1}^{2}+M_{2}^{2}\right) \hat{t}+2 \hat{t}^{2}+\hat{s}\left(2 \hat{t}-\left(M_{1}-M_{2}\right)^{2}\right)\right. & \text { fermion } \\ \left(M_{1}^{2} M_{2}^{2}-\left(M_{1}^{2}+M_{2}^{2}\right) \hat{t}+\hat{t}^{2}+\hat{s} \hat{t}\right) & \text { scalar }\end{cases}
$$


where

$$
V_{A}^{2}= \begin{cases}3\left(Q_{q} Q_{X} \frac{e^{2}}{\hat{s}}+g_{q_{A}} g_{X} \frac{g_{2}^{2} / c_{\mathrm{W}}^{2}}{\hat{s}-M_{Z}^{2}}\right)^{2} & \text { for } q \bar{q} \rightarrow X X^{*} \\ 3 w_{X}\left(\frac{g_{2}^{2}}{\hat{s}-M_{\mathrm{W}}^{2}}\right)^{2} & \text { for } u \bar{d} \rightarrow X_{1} X_{2}\end{cases}
$$

and $g=T_{3}-s_{\mathrm{W}}^{2} Q$ is the $Z$ coupling, $A=\{L, R\}$. So far we considered the case of a $\mathrm{SU}(2)_{L}$ singlet: in such a case one has $w_{X}=0$. Otherwise $w \neq 0$ if $A=L$ and $T_{3}\left(X_{1}\right)-T_{3}\left(X_{2}\right)= \pm 1: w_{X}=1$ if $X$ is a weak doublet; $w_{X}=2$ if $X$ is a weak triplet. The resulting $p p$ cross section is plotted in figure 6 and grows as $N Q^{2}$. As well known, the cross section for pair production of scalars (left) is $p$-wave suppressed and about one order of magnitude smaller than the fermion pair production cross section (right).

The experimental bounds on such cross sections depend on how $X$ decays. A large variety of possibility exists; furthermore gauged $\mathrm{SU}(N)$ could lead to 'quirk' phenomena [39]. Heavy leptons tend to give easily detectable signals, potentially giving limits as strong as the present inverse luminosity $L, \sigma \lesssim$ few $/ L \approx \mathrm{fb}$. In such a case, figure 6 implies that new fermions and (to a lesser extend) new scalars with large multiplicities and/or large charges are already excluded, if their masses are around few hundred $\mathrm{GeV}$ : if the particles are long-lived and don't decay inside the detector, the limits are 660 to $785 \mathrm{GeV}$ for electric charges from 2 to 6 [40]. However, if the particles decay inside the detectors, there are no model independent limits, and the general constraints on quasi-degenerate EW-multiplet are around $200-400 \mathrm{GeV}[41]$.

\subsection{Dark matter}

It is interesting to consider the case where $X$ lies in a $\mathrm{SU}(2)_{L}$ multiplet that contains, as lightest component, a neutral state that can be a Dark Matter candidate. At colliders Dark Matter can be seen as missing energy. If the $\mathrm{SU}(2)_{L}$ multiplet is quasi-degenerate (Minimal Dark Matter limit [42]), the decay products that allow to tag the event become soft and can be missed. One needs to rely on initial state radiation, which can give an extra jet or photon or $Z$, but with a smaller cross section, such that the signal can easily be below the SM backgrounds. In this situation a large multiplicity of light $X$ particles $\left(M \lesssim M_{S} / 2\right)$ becomes allowed.

The thermal freeze-out cosmological Dark Matter abundance is reproduced when the $s$-wave DM (co)annihilation cross-section equals to $\sigma_{0}=\sigma_{\text {cosmo }} \approx 1 /(22 \mathrm{TeV})^{2}$. In the Minimal Dark Matter limit the $\sigma_{0}$ induced by SM gauge interactions is given by

$$
\sigma_{0}=\frac{\sum_{R} d_{R}^{2} \sigma_{0}(R)}{\left(\sum_{R} d_{R}\right)^{2}}
$$

where [42]

$$
\sigma_{0}(n, Y)= \begin{cases}\frac{g_{2}^{4}\left(2 n^{4}+17 n^{2}-19\right)+4 Y^{2} g_{Y}^{4}\left(41+8 Y^{2}\right)+16 g_{2}^{2} g_{Y}^{2} Y^{2}\left(n^{2}-1\right)}{1024 \pi n c M_{R}^{2}} & \text { fermion } \\ \frac{g_{2}^{4}\left(3-4 n^{2}+n^{4}\right)+16 Y^{4} g_{Y}^{4}+8 g_{2}^{2} g_{Y}^{2} Y^{2}\left(n^{2}-1\right)}{256 \pi c n M_{R}^{2}} & \text { scalar }\end{cases}
$$


We have considered a multiple set of MDM representations $R$ that fill a $n$-dimensional representation of $\mathrm{SU}(2)_{L}$ with hypercharge $Y$. Their number of degrees of freedom is $d_{R}=$ $2 c n$ (scalar) or $d_{R}=4 c n$ (fermion) where $c=1 / 2$ (1) for a real (complex) representation. Taking into account that extra annihilations mediated by $S$ are typically subdominant [3], $N$ degenerate scalar doublets with $Y=1 / 2$ reproduce the observed DM abundance if their mass is $M=540 \mathrm{GeV} / \sqrt{N}$, which is lighter than $M_{S} / 2$ (providing decay channels for $S$ ) for $N \geq 2$. Such doublets predict extra decays $S \rightarrow \gamma Z, Z Z, W^{+} W^{-}$at an acceptable level [3]. This shows that a consistent scenario can be obtained. On the other hand, fermionic doublets or higher $\mathrm{SU}(2)_{L}$ multiplets such as triplets cannot reproduce the DM abundance unless they have a very large multiplicity $N$. For example, for $N$ real representations which transform as a $n$ under $\mathrm{SU}(2)_{L}$, the terms proportional to $g_{2}^{4}$ dominate the cross section giving

$$
\frac{\sigma_{0}(n, Y)}{\sigma_{\text {cosmo }}} \sim\left(\frac{440 \mathrm{GeV}}{M_{R}}\right)^{2} \frac{1}{N}\left\{\begin{array}{ll}
\frac{1}{2} n^{3}+\frac{17}{4} n-\frac{19}{4 n} & \text { fermion } \\
n^{3}-4 n+\frac{3}{n} & \text { scalar }
\end{array} .\right.
$$

If $\mathrm{SU}(N)$ is gauged its vectors could form quasi-stable Dark Matter [43].

\subsection{Precision observables}

Given that it is difficult to directly detect quasi-degenerate Dark Matter weak multiplets at LHC, it is interesting to explore how they indirectly affect precision data.

New scalars or fermions with hypercharge $Y$ and mass $M_{X}$ belong to the class of 'universal new physics' that affects precision data measurable at colliders with energy $\sqrt{s} \ll M_{X}$ only trough the $\mathcal{S}, \mathcal{T}, \mathcal{W}, \mathcal{Y}$ parameters [44]. We assume that these particles are not coupled to the SM Higgs doublet, so that the $\mathcal{S}$ and $\mathcal{T}$ parameters are not affected. On the other hand, the $\mathcal{Y}$ and $\mathcal{W}$ parameters receive the following contributions [45]

$$
\begin{gathered}
\mathcal{Y}=\sum_{s} \Delta b_{Y}^{(s)} \frac{\alpha_{Y}}{40 \pi} \frac{M_{\mathrm{W}}^{2}}{M_{s}^{2}}+\sum_{f} \Delta b_{Y}^{(f)} \frac{\alpha_{Y}}{20 \pi} \frac{M_{\mathrm{W}}^{2}}{M_{f}^{2}} \\
\mathcal{W}=\sum_{s} \Delta b_{2}^{(s)} \frac{\alpha_{2}}{40 \pi} \frac{M_{\mathrm{W}}^{2}}{M_{s}^{2}}+\sum_{f} \Delta b_{2}^{(f)} \frac{\alpha_{2}}{20 \pi} \frac{M_{\mathrm{W}}^{2}}{M_{f}^{2}}
\end{gathered}
$$

where $\Delta b_{Y}^{(s)}=d_{R} Y^{2} / 6$ and $\Delta b_{Y}^{(f)}=d_{R} Y^{2} / 3$ are the usual contributions to the hypercharge beta-function coefficients coming from each scalar and fermion. $\Delta b_{2}^{(s)}$ and $\Delta b_{2}^{(f)}$ are the analogous coefficients for the $\mathrm{SU}(2)_{L}$ beta functions.

The present experimental bound, $|\mathcal{Y}| \lesssim 210^{-3}$ [44], implies $\sum_{R} d_{R} Y^{2} \lesssim$ $1500\left(M_{R} / 375 \mathrm{GeV}\right)^{2}$, which is too weak to have a significant impact on our present analysis. Comparable limits on this kind of effects can be obtained from the differential $p p \rightarrow \mu^{+} \mu^{-}$ cross section at LHC at large invariant mass [46].

A future circular collider operating at the $Z$ peak can measure $\mathcal{W}, \mathcal{Y}$ with improved accuracy. According to [47-49], a precision of $10^{-6}$ on $\sin ^{2} \theta_{\mathrm{W}}$ (the effective mixing angle defined trough $Z$ couplings) is a reasonable goal. The theoretical uncertainty can be brought down to the same level, expect for the uncertainty coming from $\alpha_{\mathrm{em}}\left(M_{Z}\right)$, which 
presently is $1810^{-6}$ [47-49]. A future circular collider could reduce this uncertainty by a factor of 3 [50]. In any case this would be the dominant constraint on $\mathcal{Y}$ and $\mathcal{W}$, given that

$$
\frac{\delta \sin ^{2} \theta_{\mathrm{W}}}{\sin ^{2} \theta_{\mathrm{W}}}=\frac{\sin ^{2} \theta_{\mathrm{W}} \mathcal{W}+\cos ^{2} \theta_{\mathrm{W}} \mathcal{Y}}{\sin ^{2} \theta_{\mathrm{W}}-\cos ^{2} \theta_{\mathrm{W}}}
$$

The measurement of $\sin ^{2} \theta_{\mathrm{W}}$ with a total precision of $10^{-5}$ would determine $\mathcal{Y}$ with a $\pm 310^{-5}$ precision, if $\mathcal{W}=0$. This would give a strong test for the considered scenarios by demanding $\sum_{R} d_{R} Y^{2} \lesssim 20\left(M_{R} / 375 \mathrm{GeV}\right)^{2}$. If both $\mathcal{Y}$ and $\mathcal{W}$ are non-vanishing, $\sin ^{2} \theta_{\mathrm{W}}$ will restrict them to lie in a band, that becomes a long ellipse taking into account the other measurements. The measurement of $\sin ^{2} \theta_{\mathrm{W}}$ will be performed with high statistics during the measurements of the $\mathrm{Z}$ resonance line-shape parameters, which represents the first step in the planned baseline program [47-49]. We estimate that the precision $\delta \sin ^{2} \theta_{\mathrm{W}} \sim 10^{-5}$ will be enough to extensively probe the parameter space favored by the claimed $\gamma \gamma$ excess.

At LEP, the LEP2 run above the $Z$ peak measured $\mathcal{W}$ and $\mathcal{Y}$ as well as the $Z$-peak LEP1 run [44], because these parameters give corrections that increase with the collider energy, e.g.

$$
\frac{\sigma\left(e^{+} e^{-} \rightarrow \mu^{+} \mu^{-}\right)}{\sigma\left(e^{+} e^{-} \rightarrow \mu^{+} \mu^{-}\right)_{\mathrm{SM}}}=1-(0.67 \mathcal{W}+1.33 \mathcal{Y}) \frac{s}{M_{\mathrm{W}}^{2}} \quad \text { for } s \gg M_{\mathrm{W}}^{2} .
$$

Similarly, we estimate that an $e^{+} e^{-}$collider operating at higher energy $\sqrt{s}$ (around the $W^{+} W^{-}, Z h$ and $t \bar{t}$ thresholds) can measure $\mathcal{W}, \mathcal{Y}$ with $\pm 0.310^{-4}$ accuracy [51].

Furthermore, processes such as $e^{+} e^{-} \rightarrow \gamma Z$ can probe the anomalous $\gamma \gamma Z, \gamma Z Z$, etc vertices generated by a loop of heavy charged fermions or scalars.

\section{Conclusions}

We computed the maximal value of the width into $\gamma \gamma$ of a neutral scalar $S$ with mass $M_{S}$.

In section 2 we considered the effect of a loop of charged fermions with a Yukawa coupling $y$ to $S$. Perturbativity of $y$ was quantified by computing the scale at which $y$ or any other coupling, renormalised to higher energy, hits a Landau pole. We also impose meta-stability bounds on the $S$ potential. Figure 1 shows the maximal $\Gamma_{\gamma \gamma}$ as function of the Landau pole scale.

In section 3 we considered the effect of a loop of charged scalars with a cubic coupling to $S$. A large cubic does not lead to Landau poles, but it is, however, limited by vacuum (meta)stability and perturbativity in a way that depends on dimensionless quartic couplings, which are again subject to perturbativity bounds. Meta-stability was computed considering the multi-field critical bounce. Figure 4 shows the maximal $\Gamma_{\gamma \gamma}$ as function of the Landau pole scale. The result is similar to the fermionic case.

In both the fermionic and the scalar case we allowed for $N$ states and considered the possibility that a new $\mathrm{SU}(N)$ gauge symmetry acts on them. The maximal value of $\Gamma_{\gamma \gamma}$ allowed by perturbativity becomes qualitatively larger if either the Yukawa coupling $y$ or the scalar cubic, in their renormalization group evolution, can approach an infra-red fixed point. In such a case their maximal size is no longer controlled by Landau poles, but by 
the new gauge coupling $g$, which can be large. Non-perturbative models discussed in the literature $[3,19-24]$ are recovered in the limit where the new gauge coupling $g$ becomes non-perturbative around $M_{S}$.

In section 4 we considered the connection with Dark Matter, finding that $N \gtrsim 2$ scalar doublets with mass $M \lesssim M_{S} / 2$ can thermally reproduce the cosmological DM abundance. If they are quasi-degenerate, it becomes difficult to see them at hadronic colliders. We discussed how precision measurements can help in indirectly probing them.

\section{Acknowledgments}

This work was supported by the ERC grant NEO-NAT. We thank Antonello Polosa for useful discussions.

Open Access. This article is distributed under the terms of the Creative Commons Attribution License (CC-BY 4.0), which permits any use, distribution and reproduction in any medium, provided the original author(s) and source are credited.

\section{References}

[1] ATLAS collaboration, Search for resonances decaying to photon pairs in $3.2 \mathrm{fb}^{-1}$ of $p p$ collisions at $\sqrt{s}=13 \mathrm{TeV}$ with the ATLAS detector, ATLAS-CONF-2015-081, CERN, Geneva Switzerland (2015).

[2] CMS collaboration, Search for new physics in high mass diphoton events in proton-proton collisions at $\sqrt{s}=13 \mathrm{TeV}$, CMS-PAS-EXO-15-004, CERN, Geneva Switzerland (2015).

[3] R. Franceschini et al., What is the $\gamma \gamma$ resonance at $750 \mathrm{GeV}$ ?, JHEP 03 (2016) 144 [arXiv: 1512.04933] [INSPIRE].

[4] S. Knapen, T. Melia, M. Papucci and K. Zurek, Rays of light from the LHC, arXiv:1512.04928 [INSPIRE].

[5] S. Fichet, G. von Gersdorff and C. Royon, Scattering light by light at $750 \mathrm{GeV}$ at the LHC, arXiv: 1512.05751 [INSPIRE].

[6] C. Csáki, J. Hubisz, S. Lombardo and J. Terning, Gluon vs. photon production of a $750 \mathrm{GeV}$ diphoton resonance, arXiv:1601.00638 [INSPIRE].

[7] F. Goertz, J.F. Kamenik, A. Katz and M. Nardecchia, Indirect constraints on the scalar di-photon resonance at the $L H C$, arXiv: 1512.08500 [INSPIRE].

[8] M. Son and A. Urbano, A new scalar resonance at $750 \mathrm{GeV}$ : towards a proof of concept in favor of strongly interacting theories, arXiv:1512.08307 [INSPIRE].

[9] E. Gabrielli, K. Kannike, B. Mele, M. Raidal, C. Spethmann and H. Veermäe, A SUSY inspired simplified model for the $750 \mathrm{GeV}$ diphoton excess, Phys. Lett. B 756 (2016) 36 [arXiv: 1512.05961] [INSPIRE].

[10] G.M. Pelaggi, A. Strumia and E. Vigiani, Trinification can explain the di-photon and di-boson LHC anomalies, JHEP 03 (2016) 025 [arXiv: 1512.07225] [INSPIRE].

[11] Q.-H. Cao, Y. Liu, K.-P. Xie, B. Yan and D.-M. Zhang, The diphoton excess, low energy theorem and the 331 model, arXiv:1512.08441 [INSPIRE]. 
[12] M. Dhuria and G. Goswami, Perturbativity, vacuum stability and inflation in the light of $750 \mathrm{GeV}$ diphoton excess, arXiv:1512.06782 [INSPIRE].

[13] J. Zhang and S. Zhou, Electroweak vacuum stability and diphoton excess at 750 GeV, arXiv: 1512.07889 [INSPIRE].

[14] A. Salvio and A. Mazumdar, Higgs stability and the 750 GeV diphoton excess, Phys. Lett. B 755 (2016) 469 [arXiv: 1512.08184] [INSPIRE].

[15] Y. Hamada, T. Noumi, S. Sun and G. Shiu, An O(750) GeV resonance and inflation, arXiv: 1512.08984 [INSPIRE].

[16] J. Elias-Miro, J.R. Espinosa, G.F. Giudice, H.M. Lee and A. Strumia, Stabilization of the electroweak vacuum by a scalar threshold effect, JHEP 06 (2012) 031 [arXiv:1203.0237] [INSPIRE].

[17] S.R. Coleman, The fate of the false vacuum. 1. Semiclassical theory, Phys. Rev. D 15 (1977) 2929 [Erratum ibid. D 16 (1977) 1248] [INSPIRE].

[18] C.G. Callan Jr. and S.R. Coleman, The fate of the false vacuum. 2. First quantum corrections, Phys. Rev. D 16 (1977) 1762 [INSPIRE].

[19] K. Harigaya and Y. Nomura, Composite models for the $750 \mathrm{GeV}$ diphoton excess, Phys. Lett. B 754 (2016) 151 [arXiv: 1512.04850] [INSPIRE].

[20] Y. Nakai, R. Sato and K. Tobioka, Footprints of new strong dynamics via anomaly, arXiv: 1512.04924 [INSPIRE].

[21] E. Molinaro, F. Sannino and N. Vignaroli, Minimal composite dynamics versus axion origin of the diphoton excess, arXiv:1512.05334 [INSPIRE].

[22] J.M. No, V. Sanz and J. Setford, See-saw composite higgses at the LHC: linking naturalness to the $750 \mathrm{GeV}$ di-photon resonance, arXiv:1512.05700 [INSPIRE].

[23] J.M. Cline and Z. Liu, LHC diphotons from electroweakly pair-produced composite pseudoscalars, arXiv:1512.06827 [INSPIRE].

[24] N. Craig, P. Draper, C. Kilic and S. Thomas, Shedding light on diphoton resonances, arXiv: 1512.07733 [INSPIRE].

[25] T.P. Cheng, E. Eichten and L.-F. Li, Higgs phenomena in asymptotically free gauge theories, Phys. Rev. D 9 (1974) 2259 [inSPIRE].

[26] B. Pendleton and G.G. Ross, Mass and mixing angle predictions from infrared fixed points, Phys. Lett. B 98 (1981) 291 [INSPIRE].

[27] R. Oehme and W. Zimmermann, Relation between effective couplings for asymptotically free models, Commun. Math. Phys. 97 (1985) 569 [inSPIRE].

[28] G.F. Giudice, G. Isidori, A. Salvio and A. Strumia, Softened gravity and the extension of the standard model up to infinite energy, JHEP 02 (2015) 137 [arXiv:1412.2769] [INSPIRE].

[29] F. Staub, SARAH, arXiv:0806.0538 [INSPIRE].

[30] F. Staub, From superpotential to model files for FeynArts and CalcHep/CompHEP, Comput. Phys. Commun. 181 (2010) 1077 [arXiv:0909.2863] [INSPIRE].

[31] F. Staub, Automatic calculation of supersymmetric renormalization group equations and self energies, Comput. Phys. Commun. 182 (2011) 808 [arXiv:1002.0840] [INSPIRE].

[32] F. Staub, SARAH 3.2: Dirac gauginos, UFO output and more, Comput. Phys. Commun. 184 (2013) 1792 [arXiv:1207.0906] [INSPIRE]. 
[33] F. Staub, SARAH 4: a tool for (not only SUSY) model builders, Comput. Phys. Commun. 185 (2014) 1773 [arXiv: 1309.7223] [INSPIRE].

[34] F. Staub, Exploring new models in all detail with SARAH, Adv. High Energy Phys. 2015 (2015) 840780 [arXiv: 1503.04200] [INSPIRE].

[35] W. Porod, SPheno, a program for calculating supersymmetric spectra, SUSY particle decays and SUSY particle production at $e^{+} e^{-}$colliders, Comput. Phys. Commun. 153 (2003) 275 [hep-ph/0301101] [INSPIRE].

[36] W. Porod and F. Staub, SPheno 3.1: extensions including flavour, CP-phases and models beyond the MSSM, Comput. Phys. Commun. 183 (2012) 2458 [arXiv:1104.1573] [INSPIRE].

[37] J.E. Camargo-Molina, B. O'Leary, W. Porod and F. Staub, Vevacious: a tool for finding the global minima of one-loop effective potentials with many scalars, Eur. Phys. J. C 73 (2013) 2588 [arXiv: 1307.1477] [INSPIRE].

[38] C.L. Wainwright, CosmoTransitions: computing cosmological phase transition temperatures and bubble profiles with multiple fields, Comput. Phys. Commun. 183 (2012) 2006 [arXiv: 1109.4189] [INSPIRE].

[39] J. Kang and M.A. Luty, Macroscopic strings and 'quirks' at colliders, JHEP 11 (2009) 065 [arXiv: 0805.4642] [INSPIRE].

[40] ATLAS collaboration, Search for heavy long-lived multi-charged particles in pp collisions at $\sqrt{s}=8 \mathrm{TeV}$ using the ATLAS detector, Eur. Phys. J. C 75 (2015) 362 [arXiv:1504.04188] [INSPIRE].

[41] ATLAS collaboration, Search for charginos nearly mass degenerate with the lightest neutralino based on a disappearing-track signature in pp collisions at $\sqrt{s}=8 \mathrm{TeV}$ with the ATLAS detector, Phys. Rev. D 88 (2013) 112006 [arXiv:1310.3675] [INSPIRE].

[42] M. Cirelli, N. Fornengo and A. Strumia, Minimal dark matter, Nucl. Phys. B 753 (2006) 178 [hep-ph/0512090] [INSPIRE].

[43] A. Soni and Y. Zhang, Hidden SU(N) glueball dark matter, arXiv:1602.00714 [INSPIRE].

[44] R. Barbieri, A. Pomarol, R. Rattazzi and A. Strumia, Electroweak symmetry breaking after LEP-1 and LEP-2, Nucl. Phys. B 703 (2004) 127 [hep-ph/0405040] [INSPIRE].

[45] G. Marandella, C. Schappacher and A. Strumia, Supersymmetry and precision data after LEP2, Nucl. Phys. B 715 (2005) 173 [hep-ph/0502095] [inSPIRE].

[46] D.S.M. Alves, J. Galloway, J.T. Ruderman and J.R. Walsh, Running electroweak couplings as a probe of new physics, JHEP 02 (2015) 007 [arXiv:1410.6810] [INSPIRE].

[47] TLeP Design Study Working Group collaboration, M. Bicer et al., First look at the physics case of TLEP, JHEP 01 (2014) 164 [arXiv:1308.6176] [INSPIRE].

[48] M. Ciuchini, E. Franco, S. Mishima, M. Pierini, L. Reina and L. Silvestrini, Update of the electroweak precision fit, interplay with Higgs-boson signal strengths and model-independent constraints on new physics, arXiv:1410.6940 [INSPIRE].

[49] J. Ellis and T. You, Sensitivities of prospective future $e^{+} e^{-}$colliders to decoupled new physics, JHEP 03 (2016) 089 [arXiv:1510.04561] [INSPIRE].

[50] P. Janot, Direct measurement of $\alpha_{\mathrm{QED}}\left(m_{Z}^{2}\right)$ at the FCC-ee, JHEP 02 (2016) 053 [arXiv: 1512.05544] [INSPIRE].

[51] A. Blondel and P. Janot, A FCCee collider can measure $\sigma\left(e^{+} e^{-} \rightarrow \mu^{+} \mu^{-}\right)$with $7.510^{-4}$ $\left(2.510^{-4}\right)\left(1.510^{-4}\right)$ fractional uncertainty at $\sqrt{s}=160 \mathrm{GeV}(240 \mathrm{GeV})(350 \mathrm{GeV})$, private communication. 